\title{
The DLC-1 tumor suppressor is involved in regulating immunomodulation of human mesenchymal stromal /stem cells through interacting with the Notch1 protein
}

Tao Na, Kehua Zhang and Bao-Zhu Yuan*

\begin{abstract}
Background: Immunomodulatory activities of human mesenchymal stromal/stem cells (hMSCs) has been widely recognized as the most critical function of hMSCs for exerting its therapeutic effects. However, the detailed mechanisms responsible for regulating the immunomodulation of hMSCs still remain largely unknown. Previous studies revealed that the Notch1 protein exerted a pro-immunomodulatory function probably through interacting with the protein(s) subjective to proteasome-mediated protein degradation. The DLC-1 protein represents a well characterized tumor suppressor subjective to proteasome-mediated degradation. However, the detailed signaling pathway of Notch1 and the involvement of DLC-1 in regulating the immunomodulation of hMSCs have not been studied before.

Methods: The transfection with cDNA or siRNA into hMSCs assisted by co-culture of hMSCs with peripheral blood mononuclear cells and small molecule inhibitors of signaling proteins, followed by immunoprecipitation, Western blotting, RT-PCR, and flowcytometry, were employed to characterize the Notch1 signaling, to identify DLC-1 as a candidate proteasome-targeted protein, and to characterize DLC-1 signaling pathway and its interaction with the Notch1 signaling, in the regulation of immunomodulation of hMSCs, specifically, the inhibition of pro-inflammatory CD4 ${ }^{+}$-Th1 lymphocytes, and the release of immunomodulatory molecule IDO1.
\end{abstract}

Statistical analysis: One-way ANOVA was utilized as a statistical tool to analyze the data presented as means \pm SEM of at least three separate experiments.

Results: The present study revealed that the Notch1-Hey1 axis, but not the Notch1-Hes1 axis, was likely responsible for mediating the pro-immunomodulatory function of the Notch1 signaling. The DLC-1 protein was found subjective to proteasome-mediated protein degradation mediated by the DDB1 and FBXW5 E3 ligases and served as an inhibitor of the immunomodulation of hMSCs through inhibiting Rock1, but not Rock2, downstream the DLC-1 signaling. The Notch1 signaling in the Notch1-Hey1 pathway and the DLC-1 signaling in the DLC-1-Rock1-FBXW5 pathway exhibited a mutual exclusion interaction in the regulation of immunomodulation of hMSCs.

(Continued on next page)

* Correspondence: fangshi0712@qq.com

The Cell Collection and Research Center, National Institutes for Food and

Drug Control, No. 2 Tiantan Xili, Dongcheng District, Beijing 100050, China 
(Continued from previous page)

Conclusions: The present study uncovers a novel function of DLC-1 tumor suppressor in regulating the immunomodulation of hMSCs. It also proposes a novel mutual exclusion mechanism between the DLC-1 signaling and the Notch1 signaling that is possibly responsible for fine-tuning the immunomodulation of hMSCs with different clinical implications in hMSCs therapy.

Keywords: Notch1 protein, Immunomodulation, E3 ubiqitin ligase, Deleted in liver cancer 1 (DLC-1), Human mesenchymal stromal /stem cells (hMSCs), Hairy/enhancer-of-split related with YRPW motif protein 1 (Hey1)

\section{Background}

Human mesenchymal stromal /stem cells (hMSCs) is a group of fibroblast-like multipotent cells existing in almost all tissues with a limited self-renewal and differentiation potential to multiple cell lineages of endoderm, mesoderm and ectoderm [1]. The hMSCs of various tissue origins also exhibit unique immunomodulatory activities, which make hMSCs the most popular cell type used in stem cell-based therapies [2, 3]. To achieve the best clinical efficacy from hMSCs therapy, it is necessary to fully understand its immunomodulation, which represents the most important quality attribute of biological effectiveness of hMSCs.

The immunomodulation of hMSCs are manifested in part by their abilities to modulate almost all immune cells, such as $\mathrm{T}$ and $\mathrm{B}$ lymphocytes, natural killer cells [4], macrophages [5], and neutrophils [6]. During modulating $\mathrm{CD}^{+} \mathrm{T}$ lymphocytes, hMSCs inhibit proliferation and activity of pro-inflammatory lymphocytes, such as Type $1 \mathrm{~T}$ helper (Th1) and Type $17 \mathrm{~T}$ helper (Th17) subpopulations and promote polarization of regulatory $\mathrm{T}$ lymphocytes (Tregs) through cell-cell interaction and/ or secretion of immunomodulatory molecules [7]. Among the key molecules, the Indoleamine 2,3-dioxygenase 1 (IDO1) protein still represents a major research interest of the immunomodulation of hMSCs [8].

IDO1 is a rate-limiting enzyme for catalizing tryptophan into kynurenine [9]. Its expression is induced by proinflammatory molecules, such as IFN- $\gamma$, TNF- $\alpha$ or IL- $1 \alpha$ [10]. The importance of IDO1 in the immunomodulation of hMSCs has been established partially through employing either IDO1 silencing or small molecule IDO1 inhibitor, i.e. 1-methyl-L-tryptophan (1-L-MT). The IDO1 inhibition can cause significant reduction of various immunomodulatory activities of hMSCs, such as the reduction of inhibiting Th1 lymphocyte proliferation or promoting Treg polarization $[8,11]$. However, even though the IDO1 activities have been characterized in substantial details, the molecular mechanisms, particularly the cell signaling pathways involved in regulating IDO1 activity, still remain largely unknown.

Among various signaling pathways, the Notch1 signaling has been previously revealed for promoting the immunomodulation of hMSCs [8]. The Notch1 signaling is activated sequentially through binding of Notch1 proteins to their ligands on surface of adjacent cells and two successive proteolytic cleavages mediated by TNF- $\alpha$ converting enzyme (TACE) and $\gamma$-secretase/presenilin complex [8]. Different types of $\gamma$-secretase inhibitors have been used as experimental tools to unveil novel functions of Notch signaling [12]. Among the most commonly used inhibitors is a small peptide inhibitor Gamma-secretase inhibitor I (GSII), which shares both structural and functional similarities with Botezomib, a proteasome inhibitor used frequently in cancer research [13]. The cleavage by $\gamma$-secretase results in release of the Notch1 intracellular domain (NICD1) from plasma membrane and the NICD1 translocation into the nucleus, where it binds the CBF1/RBP-Jk; $\mathrm{Su}(\mathrm{H}) / \mathrm{Suppres-}$ sor of Hairless; Lag-1 (CSL) protein complex and turns the complex from transcriptional repressor into transcriptional activator with consequent activation of downstream effectors of the Notch1 signaling [14].

Among the downstream effectors of Notch1, hairy and enhancer of split-1 (Hes1) and Hairy/enhancer-of-split related with YRPW motif protein 1 (Hey1) have been more intensely studied [15]. These two effectors may represent different aspects of the Notch1 signaling. For example, over-expression of Hey1, but not Hes1, induced over an 80-fold decrease in Collagen Type II Alpha 1 Chain (Col2a1) transcription in a 3-dimentional differentiation induction model, suggesting that the Notch signaling played an inhibitory role on chondrogenic differentiation, in which the Notch1-Hey-1 axis, rather than the Notch1Hes1 axis, was most likely involved [16]. In addition, Hes1 and Hey1 might be differently involved in tissue development, whereas Hes1 was involved in the development of brain, skin and adipose tissues, Hey1 was associated with the development of heart and vasculature [17]. All these findings thus suggested that different Notch1 signaling axis may mediate different functional aspects of hMSCs.

The Deleted in Liver Cancer-1 (DLC-1) protein has been established as a tumor suppressor with abilities to inhibit growth, migration, invasion and metastasis of a large variety of common cancers [18-20]. While the DLC-1 gene expresses in almost all normal tissues, it is frequently absent or dramatically down-regulated in tumor tissues mainly due to genomic deletion and/or aberrant methylation at the promoter region of the gene 
[21]. In addition, the DLC-1 protein is subjected to cytoplasmic sequestration and proteasome-mediated degradation [22, 23], which may be governed by the CUL4A-DDB1-FBXW5 E3 ubiquitin ligase complex [24].

The DLC-1 protein is a multi-domain protein comprising of a Sterile Alpha Motif (SAM) domain, a Rho GTPaseActivating Protein (RhoGAP) domain and a StAR-related lipid-transfer (START) domain in its N-terminus, middle and $\mathrm{C}$-terminus, respectively. In addition, it possesses a bipartite nuclear localizing sequence (NLS) responsible for DLC-1 protein nuclear translocation and a serine-rich region likely for regulating the NLS activity [23]. Among the major functional domains, the RhoGAP domain is highly conserved responsible for catalyzing hydrolysis of Guanosine- $5^{\prime}$-triphosphate (GTP) into guanosine diphosphate (GDP) and subsequent inactivation of small Rho subfamily proteins, such as the Ras homologous A/B/C (RhoA/ $\mathrm{B} / \mathrm{C}$ ) and Cell division control protein 42 homolog (Cdc42) proteins. A prominent downstream effector of the small Rho proteins is Rho-associated protein kinase 1/2 (Rock1/ 2 ), which transmit various activities of the Rho proteins in different types of cells [25].

In this study, we discovered a mutual exclusion crosstalk between the DLC-1 signaling and the Notch1 signaling in human umbilical-cord-derived mesenchymal stromal /stem cells (hUC-MSCs) following the search of candidate proteins subjective to proteasome-mediated protein degradation. The crosstalk detailed that the DLC-1 signaling in a way of FBXW5-DLC-1-Rock1 was inhibitory to the immunomodulation of hUC-MSCs and able to interact with the Notch1 signaling represented by the Notch1-Hey1 axis in a mutual exclusion manner, thus likely providing a finetuning mechanism in the regulation of immunomodulation of hMSCs.

\section{Methods \\ Materials}

Cells: hUC-MSCs was gifted anonymously from TuoHua Biotech company (Siping, China), where the cells were isolated and purified from Wharton's Jelly of a discarded umbilical cord. The expression of the featured surface markers of hMSCs, the differentiation potentials to osteocytes, chondrocytes and adipocytes, and microbiological safety were tested for hUC-MSCs in our laboratory. Peripheral blood mononuclear cells (PBMCs) were freshly isolated using a conventional Ficoll method [26] from whole blood of healthy donors provided anonymously from local Red Cross. All data analysis associated with the use of hUC-MSCs and PBMCs in this study was conducted anonymously. Antibodies: the antibodies against IDO1, NICD1, DDB-1 and phosphor-STAT1 at Y701 (pSTAT1) were from Cell Signaling (Danvers, MA); the antibodies against DLC-1, from BD Bioscience (Franklin Lakes, NJ); the antibodies against ubiquitin, Hes1, Hey1,
Rock1 and Rock2, from Santa Cruz Biotechnology (Dallas, TX); the antibodies against Cullin 4A and FBXW5, from Abcam (Cambridge, MA); the antibody against $\beta$-actin, from Sigma (Milwaukee, WI); Horseradish peroxidase (HRP)-conjugated anti-mouse or anti-rabbit secondary antibodies, from GE Healthcare (Piscataway, NJ). All antibodies conjugated with different fluorescent dyes were from BD Bioscience. Constructs: pIDO1-Luc, pcDNA3.1DLC-1, -DLC-1- $6622, \quad$-DLC-1-662, -DLC-1-R718E containing a R718E point mutation in RhoGAP domain, and -DLC-1-RhoGAP, which is the RhoGAP domain only mutant, were constructed in previous studies $[8,23]$. Chemicals: GSI-I (SCP0004) and DAPT (D5942), $\gamma$ secretase inhibitors, were purchased from Sigma Aldrich (St. Louis, MI); Bortezomib, a proteasome inhibitor, was from ChemieTek (Indianapolis, IN); Y27632, a pan-Rock inhibitor, from EMD Millipore (Darmstadt, Germany); Phorbol-12-myristate-13-acetate (PMA) was from R\&D (Minneapolis, MN), Ionomycin was from Santa Cruz Biotechnology (Dallas, TX), Brefeldin A (BFA) was from Abcam (Cambridge, MA).

\section{Detection of hMSCs surface markers}

The hUC-MSCs surface markers, i.e. CD105, CD90 and CD73, were detected by flow cytometry using BD Stemflow hMSC Analysis Kit following the procedures described previously $[8,27]$.

\section{Osteogenic differentiation}

The osteogenic differentiation of hUC-MSCs was examined by detecting the expression of Response Gene to Complement 32 protein (RGC32), an effective biomarker of osteogenesis revealed and validated in previous studies, via a real-time Polymerase chain reaction (PCR) following previously described procedures for the induction of osteogenic differentiation described previously [8].

\section{Semi-quantitative PCR}

Conventional semi-quantitative RT-PCR was employed to detect mRNA expression of Hes-1 and Hey-1 in hUCMSCs following various treatments. The expression of Glyceraldehyde 3-phosphate dehydrogenase (GAPDH) was used as an internal control for the semi-quantitation. The sets of primer sequences were: AGCACAGACCCAAGTG TGCTG and GAAGGTGACACTGCGTTGGG, for HES1; ACGAGAATGGAAACTTGAGTTCGGC and CCCA AACTCCGATAGTCCATAGCAAG, for HEY-1; ACCA CAGTCCATGCCATCAC and TCCACCACCCTGTTGC TGT, for GAPDH.

Real-time PCR with SYBR Green quantification were set up using 1/20 of each complementary DNA (cDNA) preparation in Applied Biosystems ${ }^{\oplus} 7500$ Real-Time PCR Systems (Thermo Fisher scientific, Waltham, MA). Quantitative analysis was conducted by normalizing the 
expression level of the testing gene to that of GAPDH. The primer sequences were: GAAGTTCTGGGTCCTT TCATC and GCATGGATCGTCTGTTCTAATA, for RGC32; GGGCTGTGGAGTTTGGTGTC and CTGC TTGGGTGGGTGGAG, for Runx2; GATGGATTCC AGTTCGAGTATG and AGTGACGCTGTAGGTGAA, for Collagen I; TGCCTTTCCTGTAACGTTGGA and CCACAATGTTCTCTTCCCAAG, for Osterix; GGTC ACTGATTTTCCCACGGA and TGGATGTCAGGTCT GCGAAAC, for Osteopontin; CTGACCACATCGGCTT TC and CAGATTCCTCTTCTGGAGTTTAT, for BGLAP; AGGCTGGAGAGGCGGCTAAG and TGGAAGGTGA CACTGCGTTGG, for HES1; GGATCACCTGAAAA TGCTGCATAC and CCGAAATCCCAAACTCCGATAG, for HEY1; GCCGGACACCATGATCCTAAC and GAGC CTCAATGGCATCTCTGT, for DLC-1; GTGTGAACCA TGAGAAGTATGA and TAGAGGCAGGGATGATGTT, for GAPDH.

\section{Inhibition of Th1 lymphocyte proliferation}

The effect of hUC-MSCs on inhibiting proliferation of Th1 lymphocytes from the PIB (acronym for PMA, Ionomycin and BFA)-induced PBMCs was examinedfollowing the previously reported procedures [8]. Briefly, fresh PBMCs were co-cultured with hUC-MSCs in 5:1 ratio for $18 \mathrm{~h}$ in RPMI 1640 complete medium containing 10\% FBS and then stimulated with PIB $(25 \mathrm{ng} / \mathrm{ml}$ PMA, $1 \mu \mathrm{g} / \mathrm{ml}$ Ionomycin and $10 \mu \mathrm{g} / \mathrm{ml}$ BFA) for another $5 \mathrm{~h}$. Then, the PBMCs of all testing groups were collected and stained with both PerCP-Cy5.5-conjugated CD3 and FITC-conjugated $\mathrm{CD} 8$ for $30 \mathrm{~min}$ at room temperature. The PMBCs were then fixed with $4 \%$ paraformaldehyde fix solution for $15 \mathrm{~min}$ at room temperature. After that, the PBMCs were incubated with permeabilization medium containing PE-conjugated IFN- $\gamma$ in dark for $30 \mathrm{~min}$ at room temperature. Finally, the PBMCs were washed twice with PBS, and analyzed using BD FACS Calibur flow cytometer. Using flow cytometry, the CD3 positive lymphocytes were gated first, the $\mathrm{CD} 8^{-} \mathrm{IFN}-\gamma^{+}$subpopulation was then identified as Th1 lymphocytes, the $\mathrm{CD} 3^{+} \mathrm{CD} 8^{-} \mathrm{IFN}-\gamma^{+}$cells.

\section{Western blotting}

The procedures of conventional Western blotting were followed to monitor changes in expression of relevant proteins in hUC-MSCs following various treatments [8].

Immunoprecipitation (IP) for detecting the expression of poly-ubiquitinated proteins

The Cell lysates extracted using RIPA buffer from $1.5 \times$ $10^{6}$ cells treated with GSI-I or Bortezomib were incubated with $1 \mu \mathrm{g}$ antibody of the targeted protein for IP at $4{ }^{\circ} \mathrm{C}$ overnight, then incubated with protein $\mathrm{A} / \mathrm{G}$ agarose at $4{ }^{\circ} \mathrm{C}$ for $1 \mathrm{~h}$. After washing three times at room temperature, the agarose-bound cell lysates were then analyzed by Western blotting using ubiquitin antibody.

\section{Transient cDNA or small interfering RNA (siRNA) transfection}

The procedures reported previously for either cDNA or siRNA transfection were followed for either over-expressing, or silencing the expression of the genes to be tested. Each plasmid DNA transfection was conducted using lipofectamine 2000-mediated transfection. To achieve equal transfection efficiency from different plasmid DNAs and to avoid biased results among different transfections in each individual experiment, all plasmid DNAs were extracted by the same laboratory personnel in the same time using endotoxin-free extraction kit following the same extraction and quality testing protocols. All plasmid DNAs used in each transfection experiment were carefully calculated to ensure all of them being in equal molar concentration. In addition, the transfection efficiency of each individual experiment was evaluated by Western blotting using $\beta$-actin as internal control to ensure equal amount of cells among different transfection groups were utilized in each individual experiment. The siRNA transfection was employed to silence the expression of Notch1, DLC-1, Hes-1 or Hey-1 in hUC-MSCs and the silencing effect for each target was determined by Western blotting or RT-PCR [8]. The siRNA sequences were CACCAGUUUGAAUGGUCAAtt for Notch1; AGAACAGCACCUCUGGGAUtt for DLC-1, CGAGGUGACCCGCUUCCUGtt (1\#) [28] and AGACGA AGAGCAAGAAUAAtt (2\#) [29] for Hes1, and GUGCGG ACGAGAAUGGAAAtt (1\#) and GACCGGAUCAAUAA CAGUUtt (2\#) for Hey1 [30]. The siRNA for Rock1 (sc-29, 473) and Rock2 (sc-29,474) were purchased from Santa Cruz Biotechnology (Dallas, TX). The randomly scrambled siRNA was used as negative control.

\section{Construction of Notch1 and NICD expression vector}

The pcDNA3.1-Notch1 containing full-length Notch1 cDNA was kindly provided by Dr. Jon C. Aster [31]. The cDNA of NICD1 was amplified by RT-PCR from total RNA of hUC-MSCs and constructed into the pcDNA3.1 expression vector. The primer sequences used for amplifying NICD were: 5 '-GCTCTAGAGTGCTGCT GTCCCGCAAGCG-3' and 5'-CCCAAGCTTTTCAAC TTCССТTCTCCAACATCATTTC-3', in which Xbal and HindIII restriction sites were added for subcloning.

\section{Luciferase assay}

The luciferase assay was employed for detecting IDO1 promoter activity. The pIDO1-Luc vector used for the assay was constructed and characterized in a previous study [8]. The procedures reported previously were followed for detecting IDO1 promoter activity in response to IFN- $\gamma$ in hUC-MSCs [8]. 


\section{Data analysis}

The data collected from the assays of cell viability, surface markers, luciferase-based promoter activity and Th1 lymphocyte proliferation were expressed as means \pm SEM of at least three separate experiments. Comparison between group means was assessed using one-way analysis of variance with Newman-Keuls posttest (GraphPad Prism 4.0 Software, Inc., San Diego, CA, USA). The difference with $P<0.05$ was considered significant.

\section{Results}

The treatment with GSI-I elevated DLC-1 protein level in hUC-MSCs through proteasome inhibition

Given the dual inhibitory activities of GSI-I, we speculated from the previous study that the effect of GSI-I on the immunomodulation of hUC-MSCs was likely the consequence of interaction between Notch1 and other protein(s) that were subjected to proteasome-mediated protein degradation [8]. To identify the candidate proteins interacting with Notch1, we examined expression of different proteins likely undergoing proteasome-mediated degradation in hUC-MSCs. Among the candidate proteins were Mcl-1, DLC-1 and other proteins, which were subjective to the proteasome-mediated protein degradation $[24,32]$. Through Western blotting and immunoprecipitation, we found that the GSI-I treatment significantly elevated DLC-1 protein level with an increase also seen in polyubiquitinated form of protein (Fig. 1a \& b), suggesting that DLC-1 was subjected to the proteasome-mediated degradation in hUC-MSCs.

\section{The DLC-1 protein was involved in regulating the} expression of surface markers of hUC-MSCs

Given that the Notch1 protein is involved in regulating the expression of the surface markers of hUC-MSCs, like CD73, CD90 and CD105, and osteogenic differentiation, which was measured in part by the transcription of RGC32, a surrogate marker of the osteogenesis of hUCMSCs, revealed and validated in a previous study [8], to determine a possible involvement of DLC-1 in regulating surface markers and osteogenic differentiation of hUCMSCs, we transfected hUC-MSCs with siDLC-1 in the

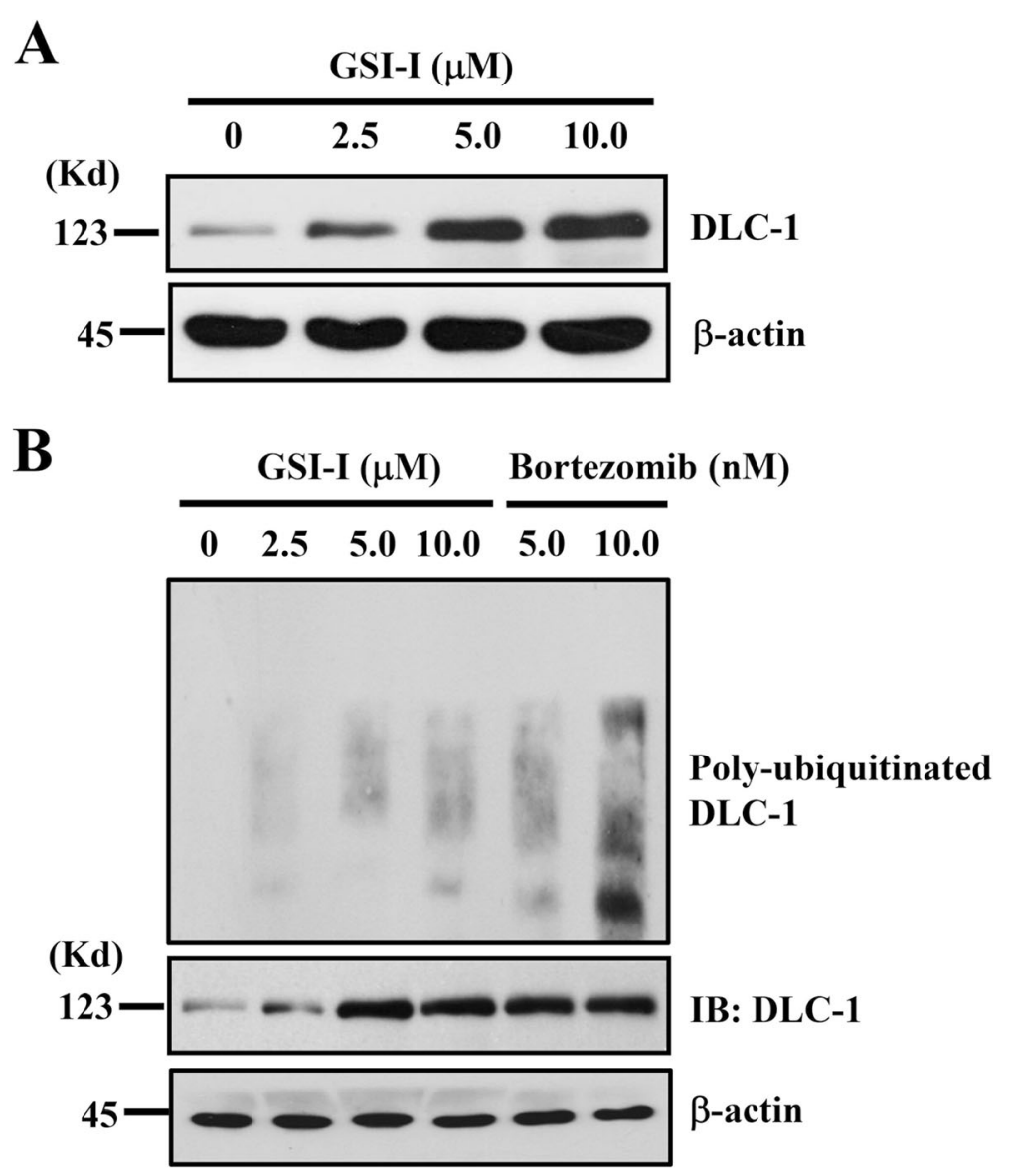

Fig. 1 The treatment with GSI-I elevates DLC-1 protein level in hUC-MSCs through proteasome inhibition. Western blotting showed a dosedependent increase in protein expression for DLC-1 at $24 \mathrm{~h}$ after GSI-I treatment (a), while the immunoprecipitation from the cell lysates using the antibodies against DLC-1 showed an increase in the polyubiquinated form of DLC-1 protein (b) 
presence of $2.5-5 \mu \mathrm{M}$ GSI-I, then tested the expression of both the surface markers and RGC32. After confirming that the silencing effect from siDLC-1 transfection (Fig. 2a), we observed that, whereas the GSI-I treatment reduced the expression of CD73, CD90 and CD105 with the most significant reduction seen in $\mathrm{CD} 105$, the siDLC-1 transfection moderately reversed the GSI-Iinduced reduction of all three surface markers (Fig. 2b). After compare the Alizarin red staining and osteogenic related gene marker determination using Realtime-PCR methods, we confirmed that RGC32 gene expression exhibited accumulatively increased along the entire osteogenic differentiation process. Therefore RGC32 is suitable for rapid determination during the early osteogenic differentiation step (Fig. 2c \& d). The siDLC-1 transfection showed no effect on the inhibitory activity of GSI-I by RGC32 expression determination (Fig. 2e). Thus suggesting that the GSI-I-induced DLC-1 elevation contributed to the GSI-I-induced reduction in surface markers, but not in osteogenic differentiation.

DLC-1 inhibited the immunomodulation of hUC-MSCs

Given that the immunomodulation of hMSCs can be represented in part by its inhibition of proliferation of Th1 lymphocyte subpopulation and IFN- $\gamma$-induced IDO1 expression [8], we transfected siDLC-1 or DLC-1

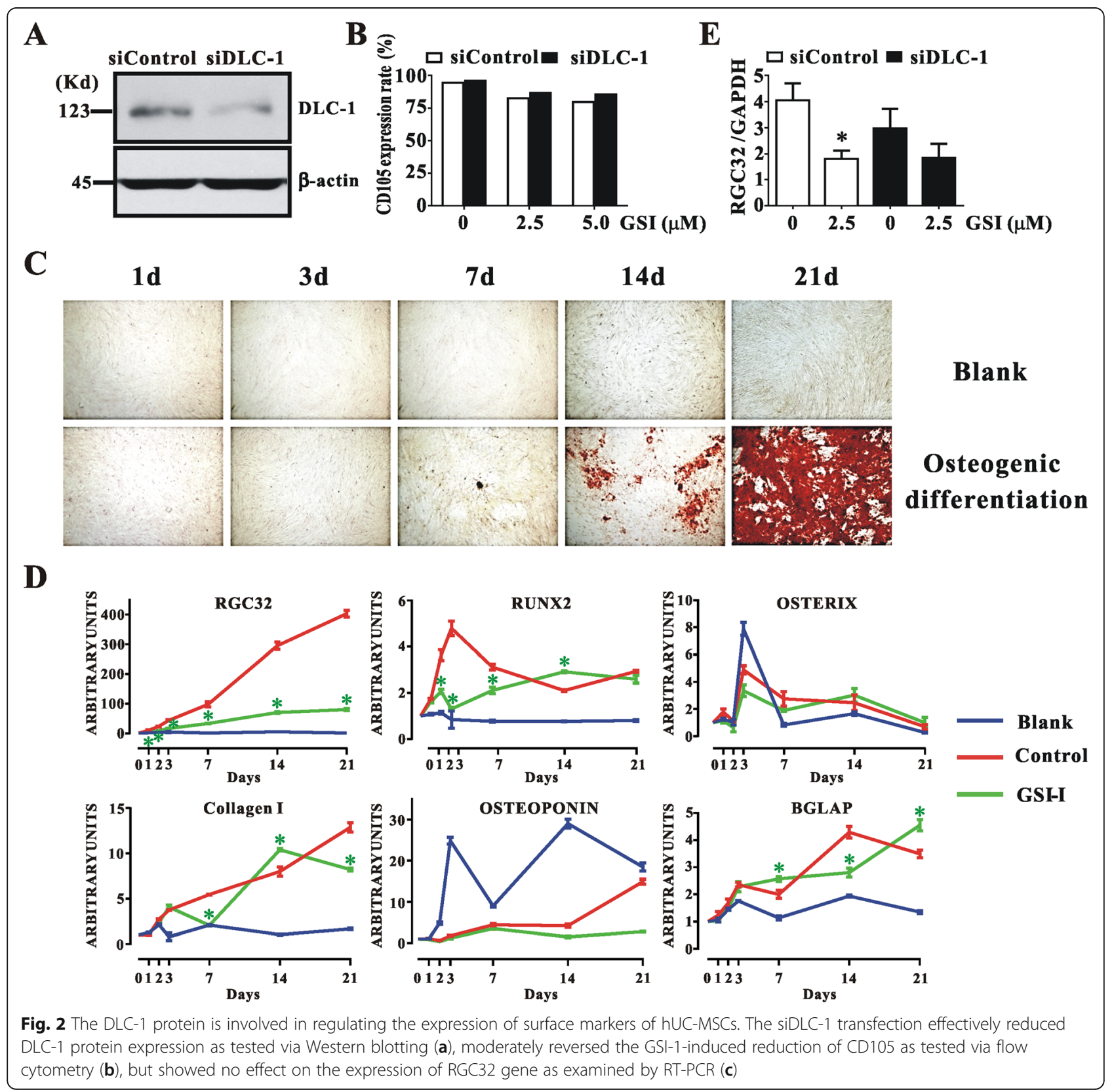


cDNA into hUC-MSCs and then examined the effect of the transfection on both Th1 proliferation and IDO1 expression. After confirming the effect of each transfection on DLC-1 expression (Fig. 3a \& b), it was observed via flow cytometry assay that, whereas the siDLC-1 transfection further enhanced the reduction of Th1 proliferation and significantly reversed the GSI-I-induced inhibition of Th1 lymphocyte proliferation as well, the DLC-1 cDNA transfection significantly reduced the inhibition of Th1 proliferation (Fig. 3a \& b). Meanwhile, through the IDO1 promoter assay, in which hUC-MSCs were cotransfected with pIDO1-Luc and siDLC-1, or pIDO1Luc and DLC- 1 cDNA for $24 \mathrm{~h}$, as followed by the treatment with $10 \mathrm{ng} / \mathrm{ml} \mathrm{IFN- \gamma}$ for another $24 \mathrm{~h}$ before measuring the luciferase activity from each transfection. It was observed that, comparing with each negative control, DLC-1 overexpression significantly reduced the IFN- $\gamma$-induced IDO1 promoter activity, whereas the DLC-1 silencing increased the promoter activity (Fig. 3c \& d), all thus suggesting for the first time that DLC-1 played an inhibitory role in the immunomodulation of hUC-MSCs.

The activity of DLC-1 inhibiting the immunomodulation of hUC-MSCs appeared to be both RhoGAP domaindependent and RhoGAP domain-independent To determine which functional domain(s) were responsible for DLC-1's activity of inhibiting the

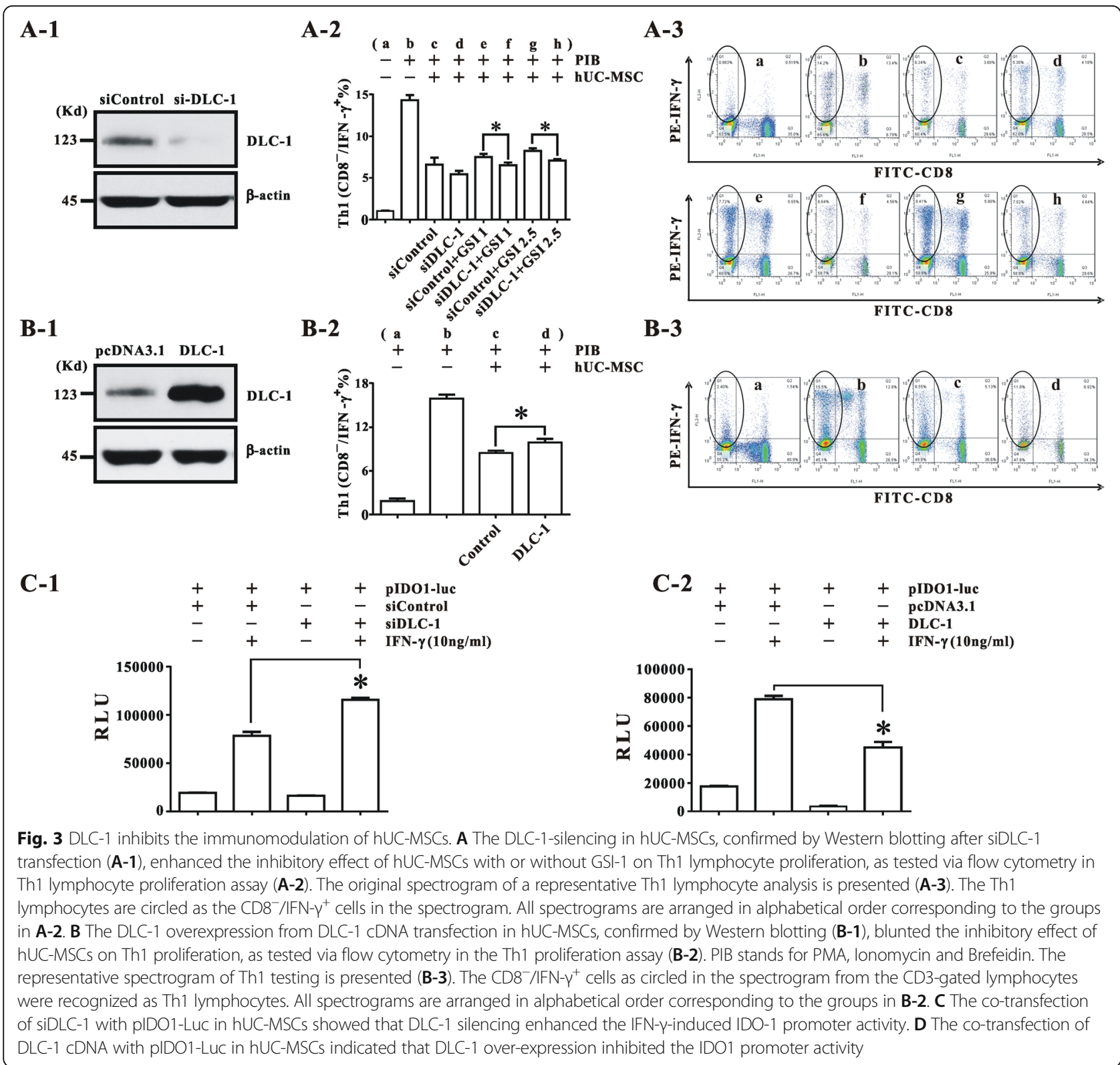




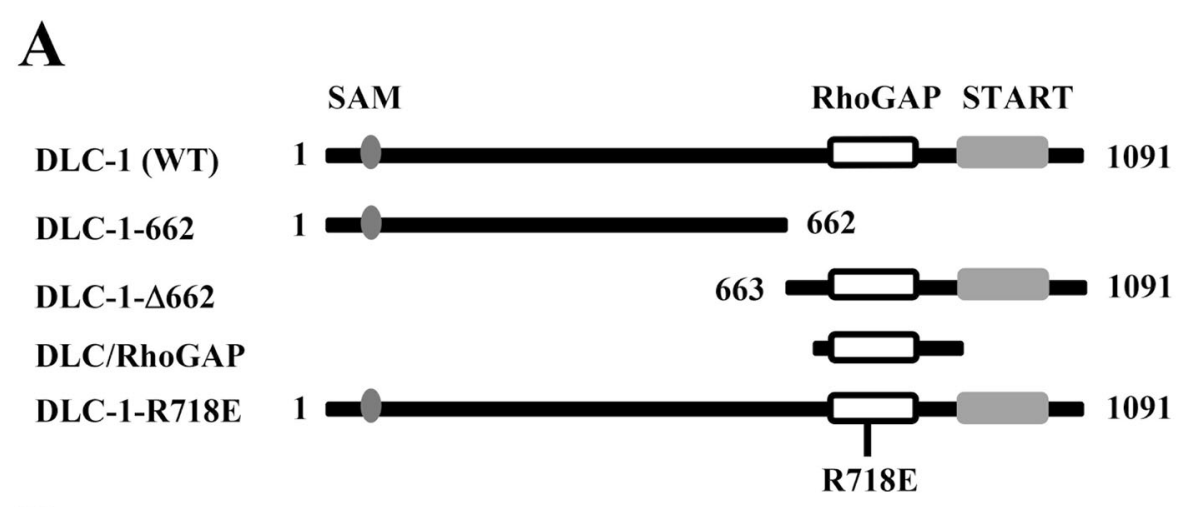

B

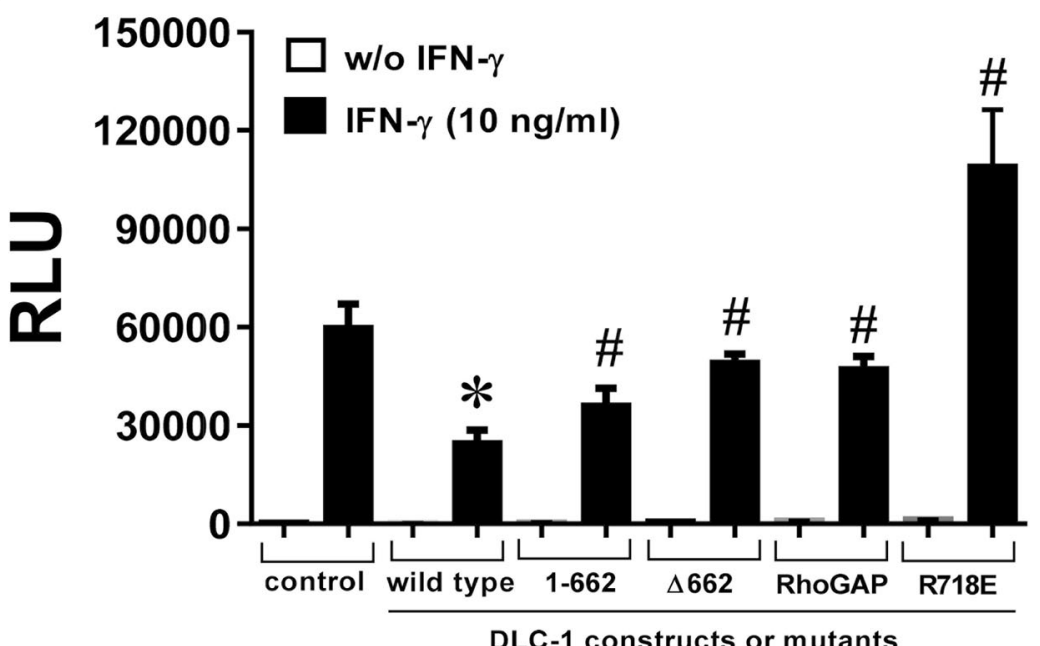

C

DLC-1 constructs or mutants

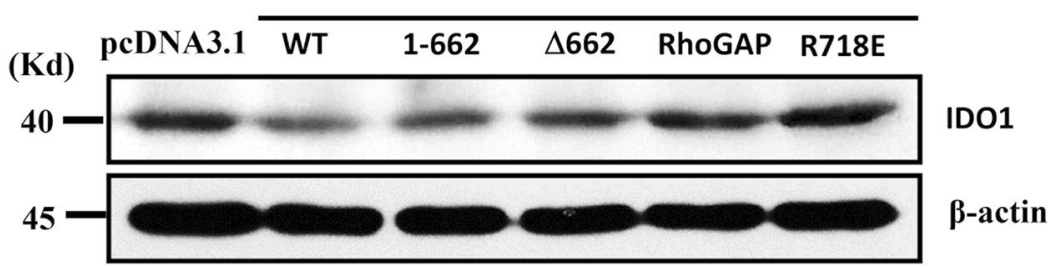

Fig. 4 The DLC-1's inhibitory effect on the immunomodulation of hUC-MSCs is both RhoGAP domain-dependent and RhoGAP domainindependent. a The schemes of all DLC-1 mutants used in this study are presented. $\mathbf{b}$ In the co-transfection of pIDO-1-Luc with the CDNA of wild type DLC-1 (wtDLC-1) or its mutants in hUC-MSCS, the wtDLC-1 showed an approximately $50 \%$ reduction of the IFN- - -induced IDO1 promoter activity, while all mutants exhibited either no effect (DLC-1-662) or even enhanced effect (DLC-1-662, $-\Delta 662,-R 714 E$ and DLC-1/RhoGAP), on the promoter activity. $\mathbf{c}$ Western bloting showed in the transfection studies that the cDNA of wtDLC-1, DLC-1-662, or DLC- $\Delta 662$ caused a significant reduction in IDO1 expression, while the CDNA of DLC-1/RhoGAP showed no effect on, and the CDNA of DLC-1/RhoGAP resulted in, a slight increase in the IDO1 expression

immunomodulation, we then tested the effect of different DLC-1 mutants, i.e. the mutant with the deletion of $\mathrm{N}$-terminus (DLC-1- $\Delta 662$ ) or C-terminus (DLC-1-622), the mutant with RhoGAP domain point mutation (DLC1-R718E), or a RhoGAP domain only mutant, in comparison with wild type DLC- 1 , on the IFN- $\gamma$-induced IDO1 promoter activity in hUC-MSCs. The schemes of all DLC-1 mutants were shown in Fig. 4a. It was observed in the co-transfection of DLC-1 or its mutant cDNAs with pIDO1-Luc that wild type DLC-1 (wtDLC1) inhibited over $50 \%$ of, the DLC-1-622 mutant showed no effect, and all other mutant showed even an increase in, the IDO1 promoter activity (Fig. 4b). In addition, the wtDLC- 1 and the DLC-1-622 and DLC-1- $\Delta 662$ mutants reduced, but the DLC-1-R718E mutant increased, the IDO1 protein expression (Fig. 4c), all thus suggesting 
that the inhibitory effect of DLC-1 on IDO1 might be both RhoGAP domain-dependent and RhoGAP domainindependent.

\section{The effect of DLC-1 in hUC-MSCs was achieved through inhibiting the Notch1 signaling}

After associating the DLC-1 protein with the immunomodulation, we were then engaged to determine the association between DLC-1 and Notch1 protein regarding the regulation of immunomodulation of hUC-MSCs. To conduct this study, we first constructed the NICD1 expression vector according to the literature [31] and validated NICD1 expression in hUC-MSCs after transfecting the vector into the cells. Next, we tried to determine the association between DLC-1 and Notch1 in regulating IDO1 promoter activity after cotransfecting DLC-1 cDNA with either siNotch1 or NICD1 cDNA. It was found that, whereas the transfection with either siNotch1 or DLC-1 cDNA alone caused a similar reduction of the promoter activity, and siNotch1 plus DLC-1 cDNA further reduced the activity (Fig. 5a). Meanwhile, it was found that, whereas the NICD1 transfection alone caused a significant increase in the promoter activity, the effect was partially reversed by DLC-1 cDNA transfection (Fig. 5b), thus suggesting the existence of an association between DLC-1 and Notch1 at least in part in the regulation of IDO1.

\section{A mutual exclusion relationship existed between DLC-1 and Notch1 in hUC-MSCs}

To further reveal the relationship between DLC-1 and Notch1, we next examined the changes of Notch1 cleavage/activation in hUC-MSCs following the transfection with siDLC-1 [8]. Interestingly, it was found that the siDLC-1 transfection caused a significant increase in both basal NICD1 and the GSI-I-reduced NICD1 (Fig. 6a). Meanwhile, the transfection with DLC-1 cDNA alone resulted in a significant reduction of NICD1 (Fig. 6b). Furthermore, it was observed in the cDNA transfection experiments that, while the DLC-1 cDNA almost completely abolished the effect of inducing IDO1 by the NICD1 cDNA, the NICD1 cDNA blocked the effect of inhibiting IDO1 expression by the DLC-1 cDNA (Fig. 6c), all thus together strongly supporting the existence of a mutual exclusion relationship in protein expression between DLC-1 and Notch1 and on the regulation of IDO1.

Regarding the likely effect of DLC-1 on NICD1 nuclear translocation, we also revealed that, whereas the siDLC-1 transfection clearly increased -NICD1 protein level in both the cytoplasm and the nucleus, the DLC-1 cDNA transfection caused a significant reduction of NICD1 protein in both compartments with a more reduction seen in the nucleus than the cytoplasm (Fig. 6d), thus indeed suggesting that DLC-1 could also reduce NICD1 protein nuclear translocation.

\section{A-1}

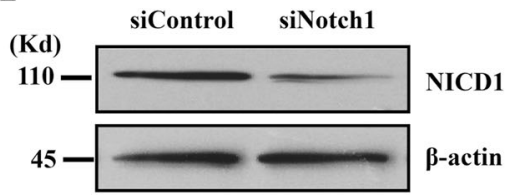

A-2

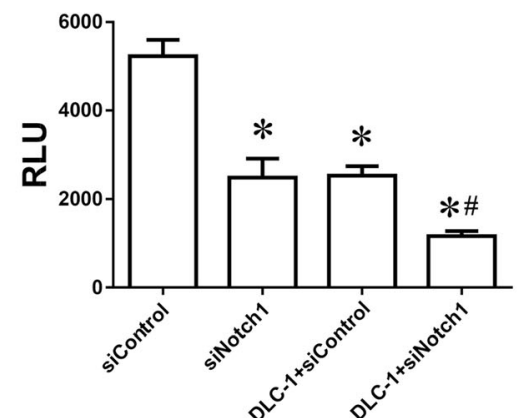

B-1

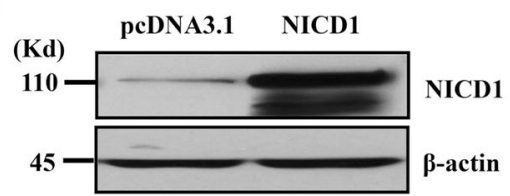

B-2

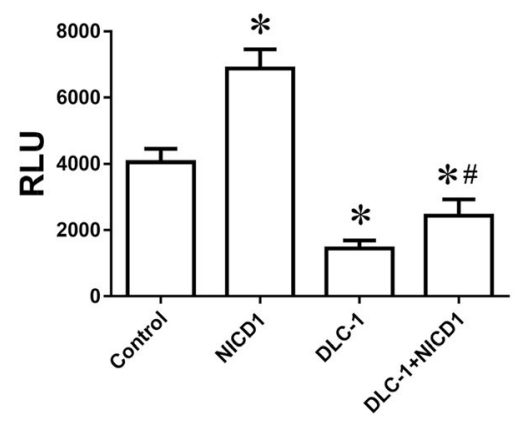

Fig. 5 The effect of DLC-1 in hUC-MSCs is achieved through inhibiting the Notch signaling. A In the IDO1 promoter assay from the cotrasnsfection of DLC-1 cDNA with siNotch1, the Notch1 silencing was confirmed by Western blotting (A-1). While DLC-1 alone or siNotch1 alone reduced, the co-transfecton further inhibited, the IDO1 promoter activity (A-2). B In the IDO1 promoter assay from the co-trasnsfection of DLC-1 CDNA with NICD1 CDNA, the NICD1 over-expression was confirmed by Western blotting (B-1). DLC-1 inhibited the NICD1-elevated IDO-1 promoter activity (B-2). The IDO1 promoter activity was measured from the transfection with plDO1-Luc followed by the treatment with IFN- $\gamma$ 


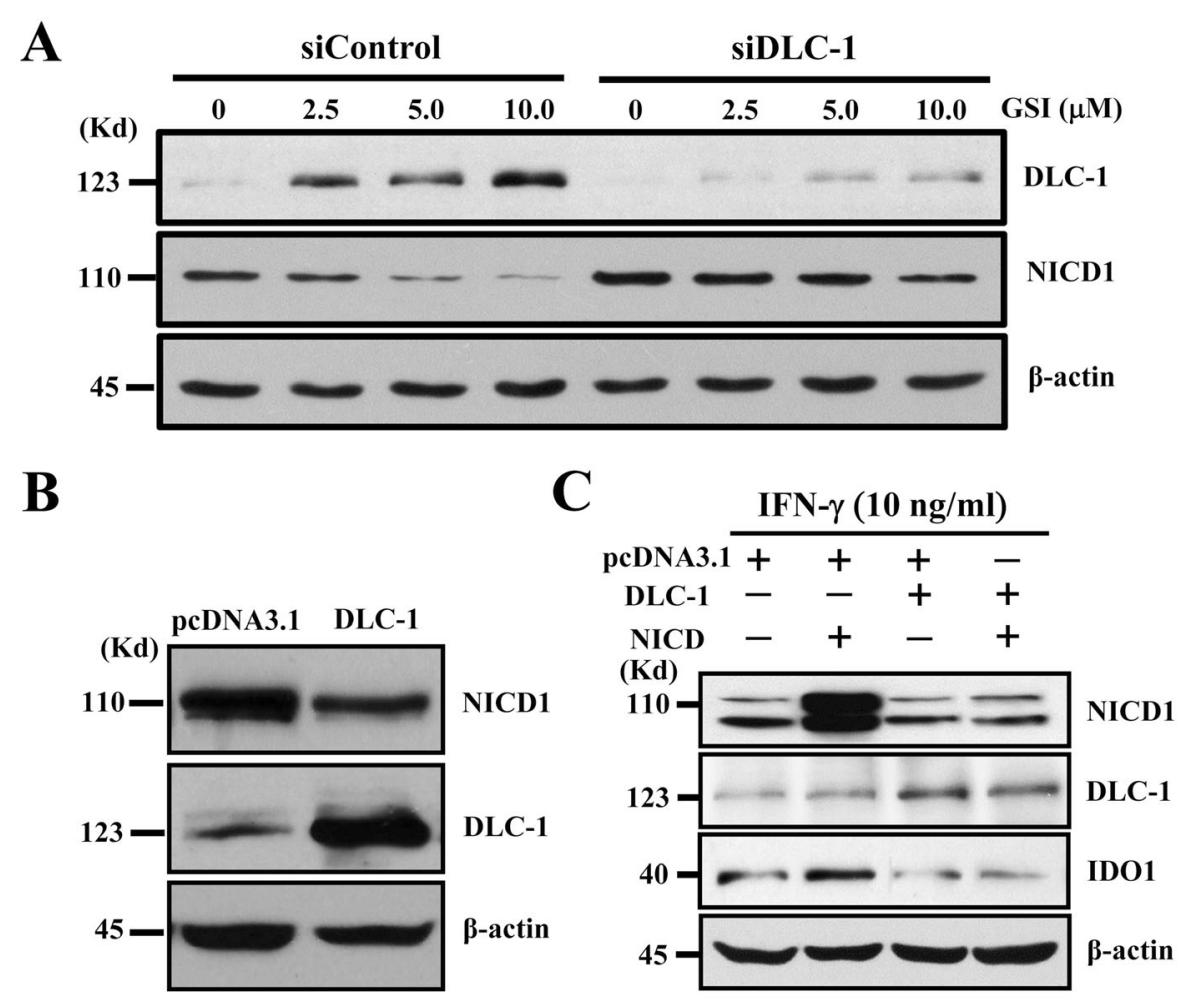

D

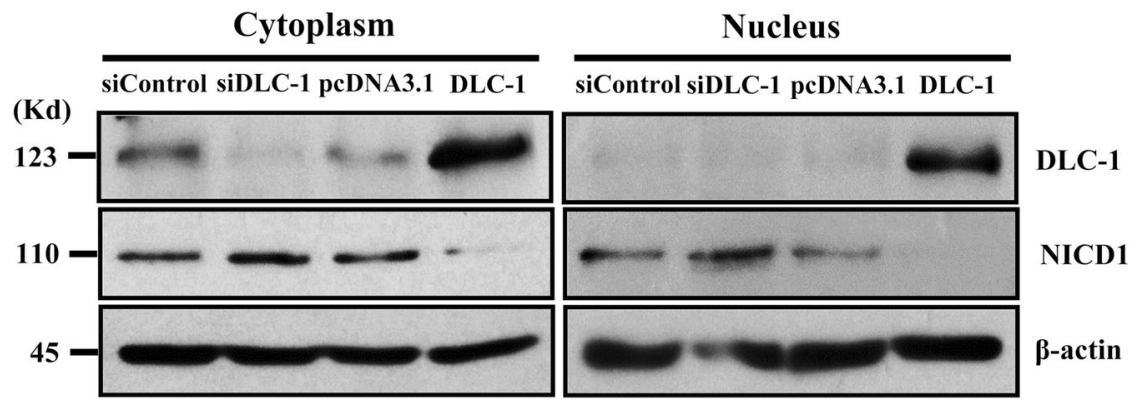

Fig. 6 A mutual exclusion relationship exists between DLC-1 and Notch1 in hUC-MSCs. Western blotting showed that the siDLC-1 transfection reduced both basal and GSI-I-induced NICD1 (a); the transfection with DLC-1 CDNA caused a significant reduction of basal NICD1 level (b); in the co-transfection studies, whereas the transfection with NICD1 CDNA alone showed an over-expression of NICD1 and increases, and the transfection with DLC-1 CDNA alone reduced, the IDO1 expression, the co-transfection of both CDNAs inhibited NICD1 over-expression and the increased expression of IDO1 (c); in analyzing the partition in cellular compartments of DLC-1 and NICD1 proteins, the siDLC-1 transfection clearly increased the NICD1 level in both the cytoplasm and the nucleus, whereas the DLC-1 CDNA transfection reduced it in both cellular

compartments with a more significant reduction seen in the nucleus than the cytoplasm (d). In all the experiments, the siControl and pcDNA3.1 were used as control in siRNA transfection and plasmid DNA transfection, respectively

The Notch-Hey 1 axis, but not the Notch-Hes 1 axis, was involved in promoting the immunomodulation of hUCMSCs

Both Hey1 and Hes1 have been well characterized as two prominent downstream effectors of the Notch signaling, but may act differently in mediating different functional aspects of the Notch signaling $[16,33]$. Therefore, it was of great interest to determine a possible distinction between these two effectors in mediating the Notch1-regulated immunomodulation of hUC-MSCs. By employing gene silencing with each specific siRNA followed by validation via RT-PCR, (Fig. 7a), we found that the Hes1 silencing caused a slight increase, but the Hey1 silencing resulted in a dramatic decrease, in the IFN- $\gamma$-induced IDO1 promoter activity (Fig. 7b). Meanwhile, it was also observed that it was the Hey1 silencing, but not Hes1 silencing, that was able to 
A

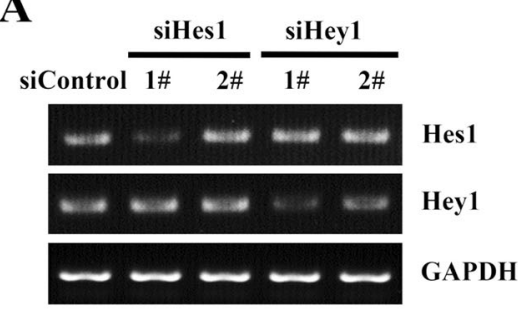

C

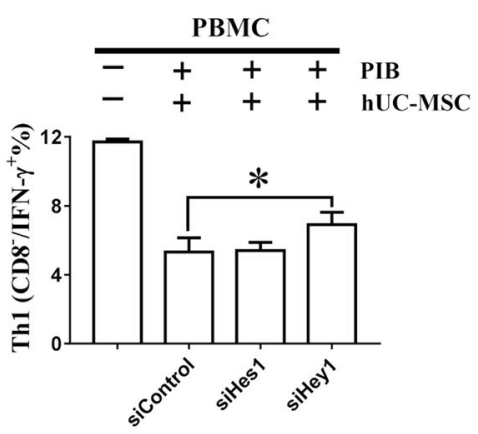

E

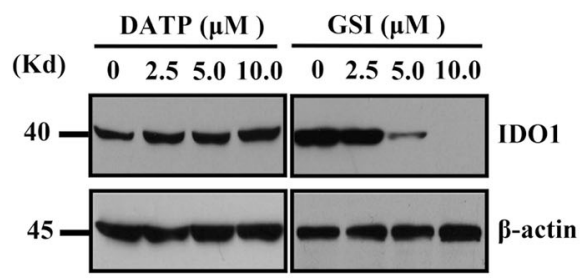

B

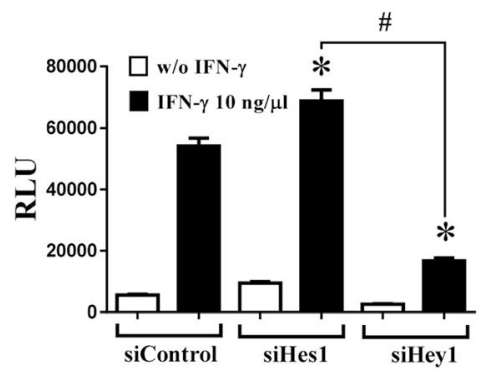

D

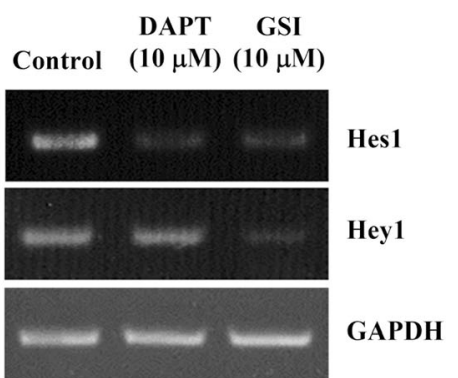

$\mathbf{F}$

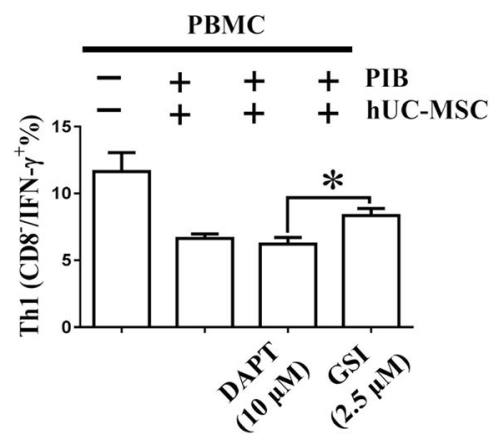

Fig. 7 DLC-1 inhibits the activity of the Notch1-Hey1 axis, but not the Notch1-Hes1 axis, in the immunomodulation of hUC-MSCs. a RTPCR validated that the transfection with siHes1 (1\#) and siHey1 (1\#) effectively inhibited the gene expression of Hes1 and Hey1, respectively. $\mathbf{b}$ The IDO1 promoter activity assay indicated that, comparing with siControl, the siHes1 transfection caused a slight increase, but the siHey1 transfection resulted in a dramatic decrease, in the IDO1 promoter activity. c The Th1 lymphocyte proliferation assay showed that the transfection with siHey1, but not siHes1, was able to significantly reduce the inhibition of Th1 proliferation by hUCMSCs. d RT-PCR showed that the treatment with GSI-I equally reduced the gene expression of both Hes1 and Hey1, whereas DAPT only reduced that of Hes1. e Western blotting showed that DAPT caused a slight increase in, but GSI-I resulted in a significant decrease in, the IDO1 expression. $\mathbf{f}$ The Th1 lymphocyte proliferation assay indicated that GSI-I reduced, but DARP exhibited no effect on, the inhibition of Th1 lymphocyte proliferation by hUC-MSCS

significantly reduce the inhibition of Th1 proliferation by hUC-MSCs (Fig. 7c), thus suggesting that it was the Notch1-Hey1 axis, but not the Notch1-Hes1 axis, that was involved in the immunomodulation of hMSCs.

Since it was reported that the Notch inhibitor DAPT exerted its inhibition more specifically on Hes1 than Hey1 [33], we then utilized DAPT as a tool in comparison with GSI-I to distinguish between Hes 1 and Hey1 in the involvement in the immunomodulation of hMSCs. It was observed through RT-PCR that, while the treatment with $10 \mu \mathrm{M}$ GSI-I equally reduced both Hes1 and Hey1 expression, the treatment with $10 \mu \mathrm{M}$ DAPT only reduced Hes1 expression, thus confirming that DAPT was a Hes1-specific inhibitor (Fig. 7d). It was next observed that, whereas the GSI-I treatment reduced both IDO1 protein expression in hUC-MSCs and the inhibition of Th1 lymphocyte proliferation by hUC-MSCs, the DAPT treatment unexpectedly increased the IFN- $\gamma$-induced IDO1 protein expression and showed no effect on Th1 lymphocyte proliferation (Fig. 7e \& $\mathrm{f}$ ), thus supporting that it was the Notch1-Hey1 axis, but not Notch1-Hes1 axis, that promoted the immunomodulation of hUC-MSCs. 
The Hey 1 protein served as a key molecule in mediating the mutual exclusion relationship between DLC-1 and Notch1

After charactering the differences between Hey1 and Hes1, we then attempted to determine whether the Hey1 protein was involved in the mutual exclusion relationship between DLC-1 and Notch1. We tested the expression of Hey1 and Hes1 genes using RT-PCR after transfection with either siDLC-1 or DLC-1 cDNA. It was found that the transfection with DLC-1 cDNA caused a slight reduction of Hes1, but a significant reduction of Hey1. In contrast, the siDLC-1 transfection resulted in a remarkable reduction of Hes1, but a significant elevation of Hey1 (Figure s1A, B \& C). On the other hand, it was observed via Western blotting that, whereas the siHes1 transfection induced an apparent increase in IFN- $\gamma$ induced IDO1 expression but a clear decrease in DLC-1 expression, the siHey1 transfection induced a remarkable decrease in IDO1 expression, but a clear increase in DLC-1 expression (Figure s1D). Therefore, all these findings together clearly demonstrated that it was Hey1, but not Hes1, that served as the key signaling molecule involved in the mutual exclusion relationship between DLC-1 and Notch1.

\section{The Notch1-Hey1 axis regulates DLC-1 protein stability} through modulating the expression of FBXW5 E3 ligase Given that the DLC-1 protein could serve as a degradation target of the CUL4A-DDB1-FBXW5 E3 complex in tumor cells [24], it was of great interest to also determine the involvement of these E3ligase proteins in regulating DLC-1 protein stability and in the relationship between DLC-1 and Notch1 in hUC-MSCs. For this purpose, we silenced the expression of each E3-ligase protein in hUC-MSCs, then determined the effect of each silencing on DLC-1 protein expression and on the immunomodulation of hUC-MSCs. Interestingly, after confirming the silencing effect of each E3 ligase by Western blotting (Figure s2A), we observed that the silencing of either DDB1 or FBXW5, but not CUL4A, significantly elevated DLC-1 protein level with a more significance seen in FBXW5 silencing. In addition, the silencing of either FBXW5 or DDB1 was accompanied by a significant reduction in IDO1 and p-STAT1 with a more significance seen again in FBXW5 silencing (Figure s2A). More interestingly, it was also observed that, comparing with DDB1 and CUL4A, the silencing of FBXW5 caused a significant reduction in the inhibition of Th1 lymphocyte proliferation (Figure s2B). These findings thus demonstrated that FBXW5 was the major E3 ligase for regulating DLC-1 protein stability and subsequent immunomodulation of hUC-MSCs.
To further pursue the possibility that the E3 ligase(s) for DLC-1 and Notch1-Hey1 signaling could be mutually regulated, we first silenced each E3 ligase and then tested its consequence on the expression of NICD1 and Hey1. We then found that, whereas the FBXW5 silencing led to a significant reduction in both NICD1 and Heyl, the silencing for DDB1 or CUL4A showed almost no such effect (Figure s2A). Next, we examined the effect of silencing Hes1 or Hey1 on the expression of all E3 ligases. It was then observed that, whereas the Hes1 silencing showed no effect on all E3 ligase proteins, the Hey1 silencing however caused a significant reduction only in FBXW5 protein, thus concluding that the Notch1-Hey1 signaling and FBXW5 could be mutually inhibitory for regulating DLC-1 protein stability and subsequent immunomodulation of hMSCs (Figure s2C).

\section{The inhibition of Rock1, but not Rock2, inhibited the immunomodulation of hUC-MSCs}

Given that the Rock $1 / 2$ proteins serve as the key effectors of the DLC-1 signaling, we next attempted to determine whether they could play a role in regulating the immunomodulation of hUC-MSCs. In the new experiments, we employed Y27632, a Rock1/2 small molecule inhibitor, to treat hUC-MSCs and then tested the effect of the treatment on IFN- $\gamma$-induced IDO1 expression and IDO1 promoter activity in hUC-MSCs. Unexpectedly, we found that the treatment with Y27632 resulted in a significant dose-dependent increase in IDO1 protein expression and promoter activity (Figure s3A \& B). To understand the seemingly contradictory effect of Y27632 on IDO1, we then tested via Western blotting the protein expression of Rock1 and Rock2, and the expression of DLC-1, NICD1, Hes1 and Hey1. Interestingly, it was found that the Y27632 treatment resulted in a dose-dependent reduction of both Rock1 and Rock2 with a much more significant reduction in Rock2. In addition, it also caused a dosedependent decrease in DLC-1, but an increase in both NICD1 and Hey1 (Figure s3A). Moreover, consistent to the increase in IDO1, the hUC-MSCs pretreated with Y27632 exhibited a significantly enhanced inhibition of Th1 lymphocyte proliferation (Figure s3C), all thus suggesting that the treatment of Y27632 in fact mimicked the activity of the Notch1 signaling in enhancing the immunomodulation of hMSCs. Considering that Y27632 is an inhibitor of both Rock1 and Rock2, we next transfected either siRock1 or siRock2 before examining the expression of the relevant proteins, thus attempting to distinguish the effect between Rock 1 and Rock 2 in the immunomodulation of hMSCs. The results showed that the transfection with siRock2, but not siRock1, achieved the same effect as Y27632 on the expression of IDO1 and DLC-1, whereas 
the siRock1 transfection resulted in a clear reduction of IDO1 and unexpectedly an increase in Rock2 (Figure s3D), suggesting that the effect of Y27632 observed above on the immunomodulation of hMSCs was attributable to the inhibition of Rock2, but not Rock1, and Rock1 and Rock2 appeared exerting differently in the regulation of the immunomodulation with Rock1 seemingly being proimmunomodulatory and Rock 2 anti-immunomodulatory. It could be further suggested that Rock 1 likely represented the inhibitory target downstream of the DLC-1 signaling in the inhibition of immunomodulation of hUC-MSCs, while Rock2 might serve as a negative feedback regulator of the DLC-1 signaling in this perspective.

\section{Discussion}

The unique immunomodulatory activities provide hMSCs a great versatility in managing various inflammatory/immune situations for treating a large variety of uncontrolled inflammatory or abnormal immune diseases. It is then reasonable to believe that the fine-tuned regulatory mechanisms yet to be identified must be always available for ensuring hMSCs to effectively sense various inflammatory environments for precisely modulating the corresponding inflammations utilizing precisely oriented regulatory capacities. Therefore, the endeavor to understand the fine-tuned mechanisms endowing such versatility is extremely important for fully appreciating the therapeutic effects of hMSCs. With a set of novel evidence, the present study demonstrates that a crosstalk between two important cell signaling represents a means of fine-tuning the immunomodulation of hMSCs.

The two signaling revealed in this study are the Notch1-Hey1 signaling and the FBXW5-DLC-1-Rock1 signaling, which regulate the immunomodulation of hMSCs in opposite directions. Whereas the Notch1Hey1 signaling promotes, the FBXW5-DLC-1-Rock1 signaling inhibits, the immunomodulation of hMSCs. More importantly, the activities of these two signaling are mutually exclusive, thus providing a means of fine-tuning the immunomodulation of hMSCs.

The mutual exclusion mechanism presented in this study is built up on a hypothesis proposed previously that some protein(s) subjective to proteasome-mediated protein degradation may antagonize the Notch1 signaling in regulating the immunomodulation of hUC-MSCs [8]. Although the search for DLC-1 represents the authors' preferential long-term interest, the result from the search indeed supports that DLC-1 serves as a good candidate fitting the hypothesis.

The DLC-1 tumor suppressor was first revealed in this study as a potential modulator of the surface markers of hMSCs, the CD105 in particular, suggesting it may be associated with overall quality of hMSCs, as CD105 is involved in multiple functions of hMSCs, including osteogenic differentiation [8, 34], angiogenesis [35], and regenerative/therapeutic potential [36]. However, the most important finding about DLC-1 in this study was the interaction between DLC-1 signaling and Notch1 signaling in regulating the immunomodulation of hMSCs.

From thoroughly analyzing the key members of each signaling and their involvement in the relationship between DLC-1 and Notch1, a sophisticated mutual exclusion mechanism between the two signaling then emerges.

On the side of the DLC-1 signaling, this study reveals for the first time that the DLC-1 tumor suppressor is able to inhibit the immunomodulation of hUC-MSCs. This novel activity of DLC-1 is supported by the evidence that the change in DLC-1 expression is directly associated with the change of IFN- $\gamma$-induced IDO1 expression and the inhibition of Th1 lymphocyte proliferation by hUC-MSCs (Fig. 3). In this regard, the activity of DLC-1 appears to be both RhoGAP domain-dependent and RhoGAP domain-independent, although the structural integrity of the protein is needed (Fig. 4). Nevertheless, this novel finding may be of great importance as hMSCs exist abundantly in tumor microenvironment and contribute to the immunosuppression for tumor progression, especially the metastatic progression [37]. If DLC-1 can inhibit the immunosuppression of hMSCs, it then exerts its tumor suppressor functions on both seeds (tumor cells) and soil (mesenchymal cells) in tumor microenvironment according to the 'seed-soil' theory of tumorigenicity [38].

When functioning in hMSCs in the association with treating inflammatory diseases, the regulation of DLC-1 by proteasome-mediated protein degradation, rather than genomic deletion and/or epigenetic changes, may provide a flexible mechanism allowing DLC-1 to exert its activity in hMSCs on the basis of necessity. In comparison, genomic deletion and/or aberrant methylation occurring frequently for DLC-1 in different human cancers represent the more rigid mechanism(s) necessary for tumor cells to achieve a complete knock-down of the tumor suppressor functions of DLC-1.

Concerning the composition of the E3 ligase complex responsible for degrading DLC-1 protein, our study suggests that the complex is composed mainly of the DDB1 and FBXW5 E3 ligases in hMSCs. However, in a set of lung cancer cells, the complex responsible for degrading DLC-1 comprises CULT4, DDB1 and FBXW5 E3 ligases all together, thus suggesting the difference existing in the composition of E3 ligases between cancer cells and hMSCs [24]. This notion is supported by a study, in which DDB1 and FBXW7, which is in the same family with FBXW5, is sufficient to form an E3 ligase complex without CUL4A for degrading MYC proteins [39], supporting that two E3 ligases, i.e. DDB1 and FBXW5, rather than three ligases are sufficient for mediating 
DLC-1 degradation in hMSCs. The discrepancy between lung cancer cells and hUC-MSCs in the composition of E3 ligase proteins may represent different regulation stringencies needed in different types of cells. The regulation for DLC-1 protein stability may be more stringent in parenchymal cells with three E3 ligases than that in mesenchymal cells using two E3 ligases, thus providing more flexibility for DLC- 1 in regulating the immunomodulation of hMSCs.

On the side of the Notch1 signaling, we first advanced our understanding about the involvement of Notch1 in immunomodulation of hMSCs by demonstrating that the Notch1 signaling diverges at the level of Hey1 and Hes1 regarding the immunomodulation of hMSCs. The new evidence revealed from the experiments employing either gene silencing or $\gamma$-secretase inhibitors GSI-I and DAPT suggest that the Notch1-Hey1 axis, rather than the Notch1-Hes1 axis, is likely involved in the immunomodulation of hMSCs (Figs. 7 and s1). As the Notch signaling possesses a large variety of functions, the distinguished roles between Hey1 and Hes1 downstream of the Notch1 signaling are of highly realistic relevance because different downstream effectors of any functionally diverse signaling need to represent different functional perspective of the signaling. More importantly, the distinction between Hey1 and Hes1 provides a sophisticated multi-level basis on the side of the Notch1 signaling for establishing the mutual exclusion relationship between Notch1 and DLC-1.

The multi-level mutual exclusion relationship between the two signaling is identified by employing a general approach, in which the function of the key molecule of each signaling was characterized for its association with the function of the opposite signaling in terms of regulating the immunomodulation of hMSCs. Through the characterization, each of the DLC-1, FBXW5 and Rock1 proteins of the DLC-1 signaling showed the ability to inhibit the expression of NICD1 and Heyl and the associated immunomodulation of hMSCs (Fig. 7). Similarly, both Notch1 and Hey1 were demonstrated for their activity of reducing the expression of DLC-1, but inducing the expression of FBXW5 (Figure s2). Interestingly, although both FBXW5 and DDB1 are directly involved in regulating DLC-1 protein stability, it is FBXW5, but not DDB1, that is directly involved in the mutual exclusion relationship, thus leading to a possibility that, within the E3 ligase complex responsible for degrading DLC-1, it is the FBXW5 protein that is more likely to act as a sensor for detecting the degradation signal released from the Notch1 signaling.

The characterization also leads to a conclusion that the mutual exclusion relationship between the DLC-1 and Notch1 signaling is formed by the intra-signaling and inter-signaling transduction. The intra-signaling transduction along the DLC- 1 signaling is in the order of FBXW5-DLC-1-Rock1. The inter-signaling transduction concerns mutual crosstalk between the two signaling; the crosstalk can be initiated at multiple levels of each signaling, transducted through the intra-signaling, released via the inter-signaling, and then sensed or received by the opposite signaling. It is thus imaginable that this type of crosstalk can provide a basis for achieving a mutual inhibition/exclusion of the two signaling regarding the regulation of the immunomodulation of hMSCs. For example, the inter-signaling transduction initiated in the Notch1 signaling can be transduced to the Hey1 protein and then released to the FBWX5 protein of the DLC-1 signaling for achieving the enhanced immunomodulation through inhibiting the DLC-1 signaling. With both inter- and intra- signaling transduction, the multi-level mutual exclusion mechanism can then provide a sophisticated automation system for fine-tunning the immunomodulation of hMSCs. The importance of this fine-tuned system is to set up a constantly dynamic control to sense and meet different needs of immunomodulation in various inflammatory environments.

With the framework about the crosstalk between the two signaling being proposed, a set of preliminary data provided in this study also suggests the existence of negative feedback control mechanism within each signaling for regulating the activity of that signaling. Within the DLC-1 signaling, the Rock2 protein may serve as a negative feedback molecule for limiting the activity of the DLC-1 signaling as the Rock2 inhibition induced by siRock2 or Y27632, which exhibits a more preferential inhibition on Rock2 than Rock1 in our experiments, inhibits DLC-1 protein expression while elevating IDO1 expression (Figure s3). Similarly, within the Notch1 signaling, the Hes1 protein may serve as a negative feedback molecule for inhibiting the Notch1 signaling as the Hes1 silencing elevates NICD1 and IDO1, but decreases DLC-1, an apparently opposite effect to the Hey1 silencing. The negative feedback control branched from the downstream effector of each signaling represents a common and effective control mechanism seen in different cell signaling pathways [40] and provides an additional level of the sophistication to the mutual exclusion crosstalk. Nevertheless, further investigation for the importance of the negative feedback mechanisms is warranted.

While more mechanistic studies are needed for fully understanding the mutual exclusion mechanism, one previous observation about the association of Caveolin-1 with the activity of $\gamma$-secretase may help interpret the signaling transduction from DLC-1 to Notch1. The DLC-1 protein can bind the Caveolin-1 protein in a START domaindependent manner [41, 42]. The binding may lead to a negative regulation of $\gamma$-secretase activity as the Caveolin1 protein is involved in the attenuation of $\gamma$-secretase- 
mediated proteolysis of Notch1 [43]. Nevertheless, further investigations are warranted to address all relevant details mediating the crosstalk between the two signaling before fully understanding the mutual exclusion crosstalk between them.

Besides the future mechanistic studies for further advancing our understanding of the mutual exclusion mechanism, the present study in fact inspires several interesting thinking which could be potentially exploited for future development of new testing methods to evaluate the quality of hMSCs or for the design of novel approaches to enhance therapeutic efficacy of hMSCs. For example, the key molecules involved in the mutual exclusion relationship may be developed as novel DLC1/Notch1-based or FBXW5/Hey1-based surrogate markers for evaluating the versatility of immunomodulation of hMSCs; Rock2-specific inhibitors may be included in novel priming/licensing strategies to enhance therapeutic efficacy of hMSCs or may be utilized as small molecule therapeutics to provoke immunomodulatory potential of endogenous hMSCs in future 'cell-free' hMSCs therapy.

\section{Conclusions}

In summary, the present study significantly advances our understanding about the regulation of the immunomodulation of hMSCs. It demonstrates for the first time that the DLC-1 tumor suppressor can function as an inhibitor of the immunomodulation of hMSCs, thus further emphasizing the importance of the DLC-1 tumor suppressor in regulating both tumor 'seed' and tumor 'soil', both of which are critical for tumor development and progression according to the 'seed-soil' theory of tumorigenicity [38]. On top of the findings about DLC-1, the present study in fact discovers a sophisticated multilevel mutual exclusion mechanism, which well fits the need of fine-tuning the immunomodulation of hMSCs. It is believed that the findings are of great potential for developing new quality evaluation biomarkers, newly designed hMSCs products or new therapeutic modalities.

\section{Supplementary Information}

Supplementary information accompanies this paper at https://doi.org/10. 1186/s12885-020-07542-5.

Additional file 1: Figure S1. Hey1, but not Hes1, mediate the mutual exclusive relationship between DLC-1 and Notch1. A-C: RT-PCR showed that the transfection with siHes 1 or siHey 1 can effectively reduce the gene expression of Hes1 (A-1) or Hey (A-2), respectively; the transfection with DLC-1 cDNA caused a slight reduction of Hes1 expression (B-1), but a significant reduction of Hey1 expression (B-2). In contrast, the siDLC-1 transfection resulted in a remarkable reduction of Hes1 expression (C-1), but a significant elevation of Hey1 expression (C-2). D. Western blotting showed that, while the siHes 1 transfection induced an apparent increase in the expression of both IDO1 and NICD1, but a clear decrease in DLC-1 expression, the siHey 1 transfection induced a remarkable decrease in the expression of both IDO1 and NICD1, but a clear increase in DLC-1 expression
Additional file 2: Figure S2. The Notch1-Hey1 axis regulates DLC-1 protein stability through modulating the expression of CULT4-DDB1FBXW5 E3 ubiquitin ligase complex. A. Western blotting confirmed that the transfection with siCULT4, siDDB1 or siFBXW5 can effectively silence the protein expression of each target gene. The silencing of both DDB1 and FBXW5, but not CUL4A, resulted in a significant increase in DLC-1 level and a significant decrease in IFN- $\gamma$-induced IDO1 and p-STAT1 level with the silencing of FBXW5 exhibiting more significant effect than that of DDB1. The FBXW5 silencing caused in a significant decrease in both NICD1 and Hey1, while the siDDB1 silencing showed only a slight reduction of NICD1 but an increase in Hey1, and the CUL4A silencing exhibits no effect on both NICD1 and Hey1. B. The Th1 lymphocyte proliferation assay showed that the FBXW5 silencing, but not the silencing of either DDB1 or CUL4A, resulted in a significant reduction of the inhibition of Th1 lymphocyte proliferation by hUC-MSCs. C. Western blotting showed that, while the Hes 1 silencing exerted no effect on the expression of CUL4A, DDB1 and FBXW5, the si-Hey1 silencing resulted in a significant reduction of FBXW5 only.

Additional file 3: Figure S3. The Y27632-induced Rock2 inhibition, but not Rock1 inhibition, promotes the immunomodulation of hUC-MSCs probably through inhibiting the DLC-1 signaling while elevating the Notch1 signaling. A. Western blotting showed that the treatment with Y27632 for $24 \mathrm{~h}$ resulted in a dose-dependent reduction of both Rock1 and Rock2 with a much more significance seen for Rock2 as accompanied by the reduction of DLC-1 and Hes1. The treatment also resulted in a significant dose-dependent increase in the IFN- $\gamma$-induced IDO1 expression, as accompanied by the increase in both NICD1 and Hey1. B. The promoter activity assay showed that the Y27632 treatment caused a dose-dependent increase in IDO1 promoter activity. C. The Th1 lymphocyte proliferation assay showed that the pretreatment with Y27632 for 24 $\mathrm{h}$ exhibited a clear increase in the inhibition of Th1 lymphocyte proliferation with the statistical significance seen in the pretreatment with $10 \mu \mathrm{M}$ Y27632. D. Western blotting showed that the silencing of Rock2, but not Rock1, exhibits the same effect as Y27632 on the expression of IDO1, DLC-1, NICD and Hey1.

\section{Abbreviations}

1-L-MT: 1-methyl-L-tryptophan; Brefeldin A: BFA; Cdc42: Cell division control protein 42 homolog; cDNA: Complementary DNA; Col2a1: Collagen Type II Alpha 1 Chain; CSL: CBF1/RBP-Jk; Su(H)/Suppressor of Hairless; Lag-1; CUL4A: Cullin-4A; DDB1: Damage Specific DNA Binding Protein 1; DLC1: Deleted in liver cancer 1; FBXW5: F-box/WD repeat-containing protein 5; GAPDH: Glyceraldehyde 3-phosphate dehydrogenase; GDP: Guanosine diphosphate; GTP: Guanosine-5'-triphosphate; Hes1: Hairy and enhancer of split-1; Hey: Hairy/enhancer-of-split related with YRPW motif protein 1; hMSC: Human mesenchymal stem/stromal cells; HRP: Horseradish peroxidase; hUC-MSCs: Human umbilical-cord-derived mesenchymal stem/stromal cells; IDO1: Indoleamine 2,3-dioxygenase 1; IL-1a: Interleukin-1 alpha; IFNY: Interferon-gamma; IP: Immunoprecipitation; NICD: Notch intracellular domain; NLS: Nuclear localizing sequence; PBMCs: Peripheral blood mononuclear cells; PCR: Polymerase chain reaction; RGC32: Response gene to complement 32 protein; Rho: Ras homologous; RhoGAP: Rho GTPaseactivating protein; Rock1/2: Rho-associated protein kinase 1/2; RT-PCR: Realtime Polymerase chain reaction; SAM: Sterile alpha motif; siRNA: Small interfering RNA; START: StAR-related lipid-transfer; STAT1: Signal transducer and activator of transcription 1; TACE: TNF-a converting enzyme; Th1: Type 1 T helper; Th17: Type 17T helper; TNFa: Tumor necrosis factor alpha; Treg: Regulatory T lymphocytes

\section{Acknowledgements}

Not Relevant

\section{Authors' contributions}

YBZ and NT designed the experiments. ZKH performed the osteogenic differentiation and Th1 lymphocyte proliferation assay and analyzed the corresponding results. NT performed other experiments and analyzed relevant results. YBT and NT wrote the paper. All authors have read and approved the manuscript. 


\section{Funding}

The research funds were granted from the National Natural Science Foundation of China (H1609-81172102) and the National Key Research and Development Program of China Stem Cell and Translational Research (2016YFA0101501) for supporting in part the studies of identifying novel biomarkers to be used for evaluating biological qualities of hMSCs through basic and applied studies. The funds were utilized to cover several key costs of this study, including the purchase of experimental consumables and key materials, data collection and analysis, and manuscript writing, submission and publication.

\section{Availability of data and materials}

The datasets generated in and/or analyzed from the current study are available from the corresponding author upon reasonable request.

\section{Ethics approval and consent to participate}

None of the cell lines used in the present study required ethics approval for their use.

\section{Consent for publication}

Not relevant.

\section{Competing interests}

The authors declare that they have no competing interests.

Received: 14 December 2019 Accepted: 20 October 2020

Published online: 04 November 2020

\section{References}

1. Jiang $Y$, Jahagirdar BN, Reinhardt RL, Schwartz RE, Keene CD, Ortiz-Gonzalez XR, Reyes M, Lenvik T, Lund T, Blackstad M, et al. Pluripotency of mesenchymal stem cells derived from adult marrow. Nature. 2002; 418(6893):41-9.

2. Squillaro T, Peluso G, Galderisi U. Clinical trials with mesenchymal stem cells: an update. Cell Transplant. 2016;25(5):829-48.

3. Montespan F, Deschaseaux F, Sensebe L, Carosella ED, Rouas-Freiss N Osteodifferentiated mesenchymal stem cells from bone marrow and adipose tissue express HLA-G and display immunomodulatory properties in HLA-mismatched settings: implications in bone repair therapy. J Immunol Res. 2014;2014:230346.

4. Ribeiro A, Laranjeira P, Mendes S, Velada I, Leite C, Andrade P, Santos F, Henriques A, Graos M, Cardoso CM, et al. Mesenchymal stem cells from umbilical cord matrix, adipose tissue and bone marrow exhibit different capability to suppress peripheral blood B, natural killer and T cells. Stem Cell Res Ther. 2013;4(5):125

5. Le Blon D, Hoornaert C, Daans J, Santermans E, Hens N, Goossens H, Berneman Z, Ponsaerts P. Distinct spatial distribution of microglia and macrophages following mesenchymal stem cell implantation in mouse brain. Immunol Cell Biol. 2014;92(8):650-8.

6. Hall SR, Tsoyi K, Ith B, Padera RF Jr, Lederer JA, Wang Z, Liu X, Perrella MA. Mesenchymal stromal cells improve survival during sepsis in the absence of heme oxygenase-1: the importance of neutrophils. Stem Cells. 2013;31(2):397-407.

7. Pianta S, Bonassi Signoroni P, Muradore I, Rodrigues MF, Rossi D, Silini A, Parolini O. Amniotic membrane mesenchymal cells-derived factors skew T cell polarization toward Treg and downregulate Th1 and Th17 cells subsets. Stem Cell Rev. 2015;11(3):394-407.

8. Na T, Liu J, Zhang K, Ding M, Yuan BZ. The notch signaling regulates CD105 expression, osteogenic differentiation and immunomodulation of human umbilical cord mesenchymal stem cells. PLoS One. 2015;10(2):e0118168.

9. Terness P, Bauer TM, Rose L, Dufter C, Watzlik A, Simon H, Opelz G. Inhibition of allogeneic $T$ cell proliferation by indoleamine 2,3-dioxygenaseexpressing dendritic cells: mediation of suppression by tryptophan metabolites. J Exp Med. 2002;196(4):447-57.

10. Meisel R, Zibert A, Laryea M, Gobel U, Daubener W, Dilloo D. Human bone marrow stromal cells inhibit allogeneic T-cell responses by indoleamine 2,3-dioxygenase-mediated tryptophan degradation. Blood. 2004;103(12):4619-21.

11. Zhang K, Na T, Wang L, Gao Q, Yin W, Wang J, Yuan BZ. Human diploid MRC-5 cells exhibit several critical properties of human umbilical cordderived mesenchymal stem cells. Vaccine. 2014;32(50):6820-7.

12. De Kloe GE, De Strooper B. Small molecules that inhibit Notch signaling Methods Mol Biol. 2014;1187:311-22.
13. Han J, Ma I, Hendzel MJ, Allalunis-Turner J. The cytotoxicity of gammasecretase inhibitor I to breast cancer cells is mediated by proteasome inhibition, not by gamma-secretase inhibition. Breast Cancer Res. 2009; 11(4):R57.

14. LaVoie MJ, Selkoe DJ. The Notch ligands, Jagged and Delta, are sequentially processed by alpha-secretase and presenilin/gamma-secretase and release signaling fragments. J Biol Chem. 2003;278(36):34427-37.

15. Iso T, Kedes L, Hamamori Y. HES and HERP families: multiple effectors of the Notch signaling pathway. J Cell Physiol. 2003;194(3):237-55.

16. Grogan SP, Olee T, Hiraoka K, Lotz MK. Repression of chondrogenesis through binding of notch signaling proteins HES-1 and HEY-1 to N-box domains in the COL2A1 enhancer site. Arthritis Rheum. 2008;58(9):2754-63.

17. Buas MF, Kabak S, Kadesch T. Inhibition of myogenesis by Notch: evidence for multiple pathways. J Cell Physiol. 2009;218(1):84-93.

18. Goodison S, Yuan J, Sloan D, Kim R, Li C, Popescu NC, Urquidi V. The RhoGAP protein DLC-1 functions as a metastasis suppressor in breast cancer cells. Cancer Res. 2005;65(14):6042-53.

19. Vigil D, Kim TY, Plachco A, Garton AJ, Castaldo L, Pachter JA, Dong H, Chen $\mathrm{X}$, Tokar B, Campbell SL, et al. ROCK1 and ROCK2 are required for non-small cell lung cancer anchorage-independent growth and invasion. Cancer Res. 2012;72(20):5338-47.

20. Wang C, Wang J, Liu H, Fu Z. Tumor suppressor DLC-1 induces apoptosis and inhibits the growth and invasion of colon cancer cells through the Wnt/beta-catenin signaling pathway. Oncol Rep. 2014;31(5):2270-8.

21. Yuan BZ, Durkin ME, Popescu NC. Promoter hypermethylation of DLC-1, a candidate tumor suppressor gene, in several common human cancers. Cancer Genet Cytogenet. 2003;140(2):113-7.

22. Luo HW, Luo QP, Yuan Y, Zhu XY, Huang SF, Peng Z, Li CL, Huang ZG, Feng WL. The intracellular stability of DLC1 is regulated by the 265 proteasome in human hepatocellular carcinoma cell line Hep3B. Biochem Biophys Res Commun. 2011;404(1):279-83.

23. Yuan BZ, Jefferson AM, Millecchia L, Popescu NC, Reynolds SH. Morphological changes and nuclear translocation of DLC1 tumor suppressor protein precede apoptosis in human non-small cell lung carcinoma cells. Exp Cell Res. 2007;313(18):3868-80.

24. Kim TY, Jackson S, Xiong Y, Whitsett TG, Lobello JR, Weiss GJ, Tran NL, Bang YJ, Der CJ. CRL4A-FBXW5-mediated degradation of DLC1 Rho GTPaseactivating protein tumor suppressor promotes non-small cell lung cancer cell growth. Proc Natl Acad Sci U S A. 2013;110(42):16868-73.

25. Riento K, Ridley AJ. Rocks: multifunctional kinases in cell behaviour. Nat Rev Mol Cell Biol. 2003;4(6):446-56.

26. Vissers MC, Jester SA, Fantone JC. Rapid purification of human peripheral blood monocytes by centrifugation through Ficoll-Hypaque and SepracellMN. J Immunol Methods. 1988;110(2):203-7.

27. Zhang T, Zheng J, Liu C, Lu Y. Expression of DLC-1 in clear cell renal cell carcinoma: prognostic significance for progression and metastasis. Urol Int. 2009;82(4):380-7.

28. Kamakura S, Oishi K, Yoshimatsu T, Nakafuku M, Masuyama N, Gotoh Y. Hes binding to STAT3 mediates crosstalk between Notch and JAK-STAT signalling. Nat Cell Biol. 2004;6(6):547-54.

29. Fan X, Mikolaenko I, Elhassan I, Ni X, Wang Y, Ball D, Brat DJ, Perry A, Eberhart CG. Notch1 and notch2 have opposite effects on embryonal brain tumor growth. Cancer Res. 2004;64(21):7787-93.

30. Kang J, Yoo J, Lee S, Tang W, Aguilar B, Ramu S, Choi I, Otu HH, Shin JW, Dotto GP, et al. An exquisite cross-control mechanism among endothelial cell fate regulators directs the plasticity and heterogeneity of lymphatic endothelial cells. Blood. 2010;116(1):140-50.

31. Chiang MY, Xu ML, Histen G, Shestova O, Roy M, Nam Y, Blacklow SC, Sacks DB, Pear WS, Aster JC. Identification of a conserved negative regulatory sequence that influences the leukemogenic activity of NOTCH1. Mol Cell Biol. 2006;26(16):6261-71.

32. Yuan BZ, Chapman J, Reynolds SH. Proteasome inhibitors induce apoptosis in human lung cancer cells through a positive feedback mechanism and the subsequent Mcl-1 protein cleavage. Oncogene. 2009;28(43):3775-86.

33. Kim WK, Meliton V, Tetradis S, Weinmaster G, Hahn TJ, Carlson M, Nelson SF, Parhami F. Osteogenic oxysterol, 20(S)-hydroxycholesterol, induces notch target gene expression in bone marrow stromal cells. J Bone Miner Res. 2010;25(4):782-95.

34. Ishiy FAA, Fanganiello RD, Kobayashi GS, Kague E, Kuriki PS, Passos-Bueno MR. CD105 is regulated by hsa-miR-1287 and its expression is inversely correlated with osteopotential in SHED. Bone. 2018;106:112-20. 
35. Fonsatti E, Altomonte M, Nicotra MR, Natali PG, Maio M. Endoglin (CD105): a powerful therapeutic target on tumor-associated angiogenetic blood vessels. Oncogene. 2003;22(42):6557-63.

36. Mark P, Kleinsorge M, Gaebel R, Lux CA, Toelk A, Pittermann E, David R, Steinhoff G, Ma N. Human mesenchymal stem cells display reduced expression of CD105 after culture in serum-free medium. Stem Cells Int. 2013;2013:698076.

37. Ling W, Zhang J, Yuan Z, Ren G, Zhang L, Chen X, Rabson AB, Roberts Al, Wang $Y$, Shi $Y$. Mesenchymal stem cells use IDO to regulate immunity in tumor microenvironment. Cancer Res. 2014;74(5):1576-87.

38. Faraji F, Eissenberg JC. Seed and soil: a conceptual framework of metastasis for clinicians. Mo Med. 2013;110(4):302-8.

39. Wong WJ, Qiu B, Nakazawa MS, Qing G, Simon MC. MYC degradation under low $\mathrm{O} 2$ tension promotes survival by evading hypoxia-induced cell death. Mol Cell Biol. 2013;33(17):3494-504.

40. Hermann-Kleiter N, Thuille N, Pfeifhofer C, Gruber T, Schafer M, Zitt C, Hatzelmann A, Schudt C, Leitges M, Baier G. PKCtheta and PKA are antagonistic partners in the NF-AT transactivation pathway of primary mouse CD3+ T lymphocytes. Blood. 2006;107(12):4841-8.

41. Du X, Qian X, Papageorge A, Schetter AJ, Vass WC, Liu X, Braverman R, Robles Al, Lowy DR. Functional interaction of tumor suppressor DLC1 and caveolin-1 in cancer cells. Cancer Res. 2012;72(17):4405-16.

42. Yam JW, Ko FC, Chan CY, Jin DY, Ng IO. Interaction of deleted in liver cancer 1 with tensin2 in caveolae and implications in tumor suppression. Cancer Res. 2006;66(17):8367-72.

43. Kapoor A, Hsu WM, Wang BJ, Wu GH, Lin TY, Lee SJ, Yen CT, Liang SM, Liao YF. Caveolin-1 regulates gamma-secretase-mediated AbetaPP processing by modulating spatial distribution of gamma-secretase in membrane. J Alzheimers Dis. 2010;22(2):423-42.

\section{Publisher's Note}

Springer Nature remains neutral with regard to jurisdictional claims in published maps and institutional affiliations.

Ready to submit your research? Choose BMC and benefit from:

- fast, convenient online submission

- thorough peer review by experienced researchers in your field

- rapid publication on acceptance

- support for research data, including large and complex data types

- gold Open Access which fosters wider collaboration and increased citations

- maximum visibility for your research: over $100 \mathrm{M}$ website views per year

At $\mathrm{BMC}$, research is always in progress.

Learn more biomedcentral.com/submissions 